\title{
Endoprosthetic Reconstruction in Limb Salvage for Malignant Bone Tumours in Children
}

\author{
Sagar Tontanahal', Gahukamble Abhay Deodas', Deeptiman James' ${ }^{1}$, Anand Kurian², Thomas Palocaren ${ }^{1 *}$ \\ 'Department of Paediatric Orthopaedics, Christian Medical College, Vellore, India \\ ${ }^{2}$ Department of Orthopaedics, Christian Medical College, Vellore, India
}

\section{Article Info}

\section{Article Notes}

Received: April 30, 2021

Accepted: June 01, 2021

\section{*Correspondence:}

*Dr. Thomas Palocaren, Department of Paediatric

Orthopaedics, Christian Medical College, Vellore, India; Email: thomaspalox@cmcvellore.ac.in.

${ }^{\oplus} 2021$ Palocaren T. This article is distributed under the terms of the Creative Commons Attribution 4.0 International License.

\section{Keywords}

Endoprosthetic reconstruction

Limb salvage

Malignant tumors

Children

\section{Abstract}

Background: The management of malignant bone tumors in children has come a long way in the past few decades. The transition from amputation to limb salvage has been made possible due to the rapid development in the diagnosis and the oncological management of these malignant tumors. However, there exist significant reservations regarding endoprosthetic reconstruction in children.

Material and methods: A mini-review was conducted of articles detailing the use of prosthetic reconstruction following tumor resection in children. The data regarding complications and functional outcomes following surgery were collected and presented.

Results: The studies reviewed reported a 5-year survival rate between $60-70 \%$. Uniform across the studies was the need for multiple surgeries when endoprosthesis was used for limb reconstruction, ranging between 2.8 -3.5 surgeries. The most common complication noted across the studies was related to soft tissue problems such as joint instability followed by structural failure of the prosthesis. Infections were noted with a frequency of $10-15$ $\%$. Studies showed successful management of limb length discrepancy with expandible prosthesis. Musculoskeletal Tumor Society (MSTS) score used to evaluate the functional outcome showed satisfactory outcomes.

Conclusion: Limb salvage surgery, with recent advances in technique and prosthesis design, is an attractive option in children with extremity malignant bone tumors. In recent time, endoprosthetic reconstruction of extremities have yielded good functional results and are well accepted by the child and the parents. The purpose of this mini-review is to shed some light on the use of endoprosthetic reconstruction in children following tumor resection with its potential benefits and drawbacks.

\section{Introduction}

The treatment of extremity malignant tumors in children has evolved tremendously over the past few years. In the early part of the $20^{\text {th }}$ century, the only curative treatment modality was amputation, offering a 5-year survival rate between 12 and $23 \%{ }^{1,2}$. In children, however, the survival rates following amputation were consistently found to be low, with studies by Campannaci and Cervellati ${ }^{3}$ showing 10-year survival rates as low as $11 \%$.

In the late $20^{\text {th }}$ century, there was a tremendous improvement in the treatment of malignant tumors using chemotherapy and radiation therapy, as evidenced by improved 5-year survival rates of up to $70 \%$. In 1979 , the study of Copeland et al. ${ }^{6}$ advocated the possible success of limb salvage surgery following wide en-bloc resection in selected cases of malignant bone tumors. 
The landmark study, which broadened the scope of limb salvage surgery, was published in 1986 by Simon and his colleagues? ${ }^{7}$. In this study, they found no difference in the 5-year survival of patients with osteosarcoma who underwent amputation or limb-salvage surgery. Following this study, there has been a paradigm shift in the trend towards limb salvage with an assumption that limb salvage is a better option both functionally and psychologically for the child and the family.

In the pediatric population, the acceptability of amputation by the child, the higher rates of reported complications and the need for further procedures to manage limb length discrepancy following physeal resection, make limb salvage challenging.

Recent advances in endoprosthetic design coupled with developments in the field of cancer treatment makes limb salvage surgery with endoprosthesis a viable option for management of extremity malignant tumors in children.

\section{Discussion}

\section{History of metallic endoprosthesis}

The idea of limb salvage surgery has undergone tremendous changes since its initiation. The idea of joint spanning with a fusion construct was the initial idea for limb salvage in children with the idea to preserve length and distal function. This concept gave way to prosthetic design intending to preserve joint function as well. It was Scales in $1976^{8}$, who revolutionized the use of endoprosthesis in limb salvage surgery in children. Scales and his colleagues from the division of Biomedical Engineering in Stanmore, UK, developed an extendible endoprosthesis which, in addition to preserving joint function, addressed the issue of limb length discrepancy following physeal excision.

Unwin and Walker ${ }^{9}$ studied the outcomes of limb salvage surgery in children between 1976 and 1992 . They noted 4 distinct designs of the expanding implants based on the growing mechanism. The first design used a worm drive screw for expanding the telescoping titanium components. This was later replaced by a ball bearing mechanism in 1982 after a growing number of mechanical failures were noted. In 1988, this design was replaced by a C collar mechanism, which required open dissection, manual traction, and placement of a C collar spacer.

All these prostheses required invasive surgical procedures to carry out lengthening. With improvement in manufacturing techniques, the design of the endoprosthesis reverted to a screw mechanism that could be controlled with an Allen wrench inserted percutaneously. This obliviated the need for open surgery thereby reducing the incidence of complications ${ }^{10}$.
The next advancement in the design of the expandable prosthesis came from the Stanmore group, described by Gupta and his colleagues in $2006^{11}$. This prosthesis has a magnetic disk within the prosthesis which is attached to a power screw through a gearbox. This prosthesis can be lengthened by a circular external drive unit, which induces a circular magnetic field, which in turn drives the power screw. This prosthesis came in three lengths which provide $50 \mathrm{~mm}, 70 \mathrm{~mm}$, and $90 \mathrm{~mm}$ of lengthening. This offered the advantage of non-invasive, predictable, and reversible lengthening without the need for additional surgical procedures thereby reducing the complications associated with endoprosthetic reconstruction.

Alternative designs of non-invasive expanding endoprosthesis have also been developed and are being used like, the Repiphysis prosthesis ${ }^{12}$. The expansion mechanism rests in the tubular portion of the prosthesis and utilizes the stored energy from compressed springs for expansion. The expansion is brought about by an externally applied magnetic field that melts the polymer tube housing the coiled spring, which releases the spring producing the force needed to expand the tubular insert.

The MUTARS (Modular Universal Tumor and Revision System) with their Xpand prosthesis target growth of the prosthesis by both mechanical and biological means. The device uses a high-frequency transmitter to control the actuator within the prosthesis and for the biological aspect of growth, it uses an intramedullary rod system which is capable of distraction osteogenesis ${ }^{13}$.

\section{Considerations in selecting a prosthesis in children}

The primary goals of tumor management are of paramount importance when considering limb salvage surgery in children. These include accurate diagnosis and staging followed by appropriate chemotherapeutic management and radiation therapy if necessary. Another important aspect to consider in children while using endoprosthesis for reconstruction is the age at which the tumor resection is being done and the expected limb length discrepancy. The course of treatment with limb salvage surgery can be prolonged, requiring multiple surgical procedures in addition to the protracted course of multiagent chemotherapy.

When considering the choice of endoprosthesis for reconstruction, the growing child can be subdivided into three categories based on estimated growth remaining at the resected physis ${ }^{13}$. The first, nearing skeletal maturity with $2 \mathrm{~cm}$ or less of growth remaining. In this subset, normal adult implants can be used with the residual limb length discrepancy being managed by an external shoe raise. Alternatively, the operated limb can be lengthened to the expected limb length at skeletal maturity using a long implant. The extent of lengthening is limited by the 
potential to induce sciatic nerve damage or loss of knee range of motion.

The second group are the ones who are expected to have a limb length discrepancy between 2 and $6 \mathrm{~cm}$. This group can be managed by one of two equally effective methods. The first, using a combination of ipsilateral lengthening using an adult implant and timely contralateral epiphysiodesis. The advantage of this being the use of adult prosthesis avoids the need for revision at skeletal maturity. The alternative being the use of a growing endoprosthesis. The advantage of this is that the ipsilateral limb can mirror the growth of the normal limb, the growth of which is affected by tumor treatment modalities. Additionally, this avoids operative intervention of the unaffected side, which may be a concern in some patients.

The final group is one far from skeletal maturity with growth of more than $8 \mathrm{~cm}$ remaining. The only option for endoprosthetic reconstruction is the use of growing endoprosthesis. The use of endoprosthesis in this subgroup has been reported in small case series and case reports. This group will require more than one growing prosthesis in addition to final conversion to an adult implant at skeletal maturity. This option is one requiring major commitment on behalf of the child, parents and the treating surgeon.

\section{Complications and failures}

The goals for surgical management of extremity sarcoma in children is get the child functioning at near normal levels. This can be achieved by restoring the range of motion and stability of the joint, maintaining distal functioning and also prevent local recurrence and infections. The five-year survival rates in various studies, which evaluated the use of endoprosthesis for limb reconstruction, was around $60-70 \%^{14,15,16}$. This might not be an accurate estimation of the survival rates as almost all of the studies had a selection bias, restricting the choice of cases to be ones without metastasis and low volume tumors.

The course of treatment with endoprosthesis is a long one and involves multiple surgeries. Studies report anywhere between an average of $2.5-8.7$ surgeries per child. This number has reduced with the improvement in surgical technique and implant design. Failure is defined as the need for revision of the prosthesis or surrounding soft tissue following the index surgery. Henderson and colleagues classified failures due to endoprosthetic reconstruction in adults into 5 groups which was later modified by the author to include complications specific to the pediatric population. It is also interesting to note that the different regions where the endoprosthesis were used showed different complication rates.

The Henderson type I complication, involving the soft tissues, was the most common observed. This complication included joint instability, tendon rupture and aseptic wound dehiscence. Studies by Schinhan et al. ${ }^{16}$ and Dotan et al. ${ }^{14}$ reported an incidence of 46 and $47 \%$ respectively. A recent study by Tsuda et al. ${ }^{17}$, which reviewed 124 children who underwent extendable endoprosthetic reconstruction for malignant tumors showed the incidence of this complications of $27 \%$. The incidence of complications also varied on the site of use of the endoprosthesis. Higher rates were observed in the proximal femur, distal femur and proximal tibia. This could be attributed differential growth between the acetabulum and proximal femur at the hip joint and stiffness and the patellar maltracking at the knee joint respectively.

The next most frequently encountered complication was structural failure (type 3 failure). This encompassed periprosthetic and prosthetic fracture, stress shielding and failure of growing mechanism. Three studies by Schinhan et al. ${ }^{16}$, Dotan et al. ${ }^{14}$ and Tsuda et al. ${ }^{17}$, which followed up a good number if patients for a significantly long-time frame, report an incidence of type 3 failure of $28 \%, 23 \%$ and 26 $\%$ respectively. Distal femoral endoprosthesis showed the greatest propensity for this type of complication. Infections (type 4 failure) were the next most common complication. The high rates of infection in these patients can be attributed to their immunodeficiency state as a consequence of the systemic chemotherapy they receive. The rates of infection as reported by various studies are in the range of $10-15$ $\%$. Several authors thereby recommend the use of systemic antibiotics during the post-operative period.

The incidence of aseptic loosening is relatively high as reported by various studies. This is attributed to the increase in canal diameter of the bone with growth of the child which thereby results in the loosening of the stem. The studies by Dotan et al. ${ }^{14}$, Tsuda et al. ${ }^{17}$ and Zou et $a .^{18}$ report the incidence of aseptic loosening as 28 $\%, 26 \%$ and $15.6 \%$ respectively. The highest incidence, amongst the different sites of use of the endoprosthesis, was seen around the knee with almost similar incidence between the distal femur and proximal tibial implants. In a systematic review by Groundland and colleagues ${ }^{19}$, they compared the outcomes of endoprosthesis in children and adults, they found that children fared well. The study also found that only the children undergoing proximal femoral endoprosthetic reconstruction were at a higher risk of failure. They also noted significantly reduced rates of subsequent amputations compared to the adult population.

\section{Outcomes}

The expandible prosthesis provide an attractive option for the management of limb length discrepancy following physeal resection in children. Various studies have shown the effectiveness of the expanding endoprosthesis in this 
respect. In studies by Tsuda et al. ${ }^{17}$ and Zou et al. ${ }^{18}$, the average lengthening carried out was $39.5 \mathrm{~mm}$ and $42 \mathrm{~mm}$ respectively. Study by Schinhan et al. ${ }^{16}$ and Groundland et al. ${ }^{19}$ reported greater extent of lengthening in their case series with an average lengthening of $70.5 \mathrm{~mm}$ and 84.8 $\mathrm{mm}$ respectively. In all these studies, the majority of the cases had a limb length discrepancy of less than $2 \mathrm{~cm}$ at final follow up, which can easily be managed by footwear modifications.
Studies report good functional outcomes following endoprosthesis reconstruction in children. The studies utilize the Musculoskeletal Tumor Society (MSTS) score to evaluate the functional outcome which encompass pain, emotional acceptance, function, use of ambulatory support, walking ability and gait. Studies report an average MSTS score of around $82-83 \%{ }^{14,15,16,17,18,19}$. The score varied minimally depending on the site of the endoprosthesis use, with best scores obtained for the proximal tibial prosthesis.

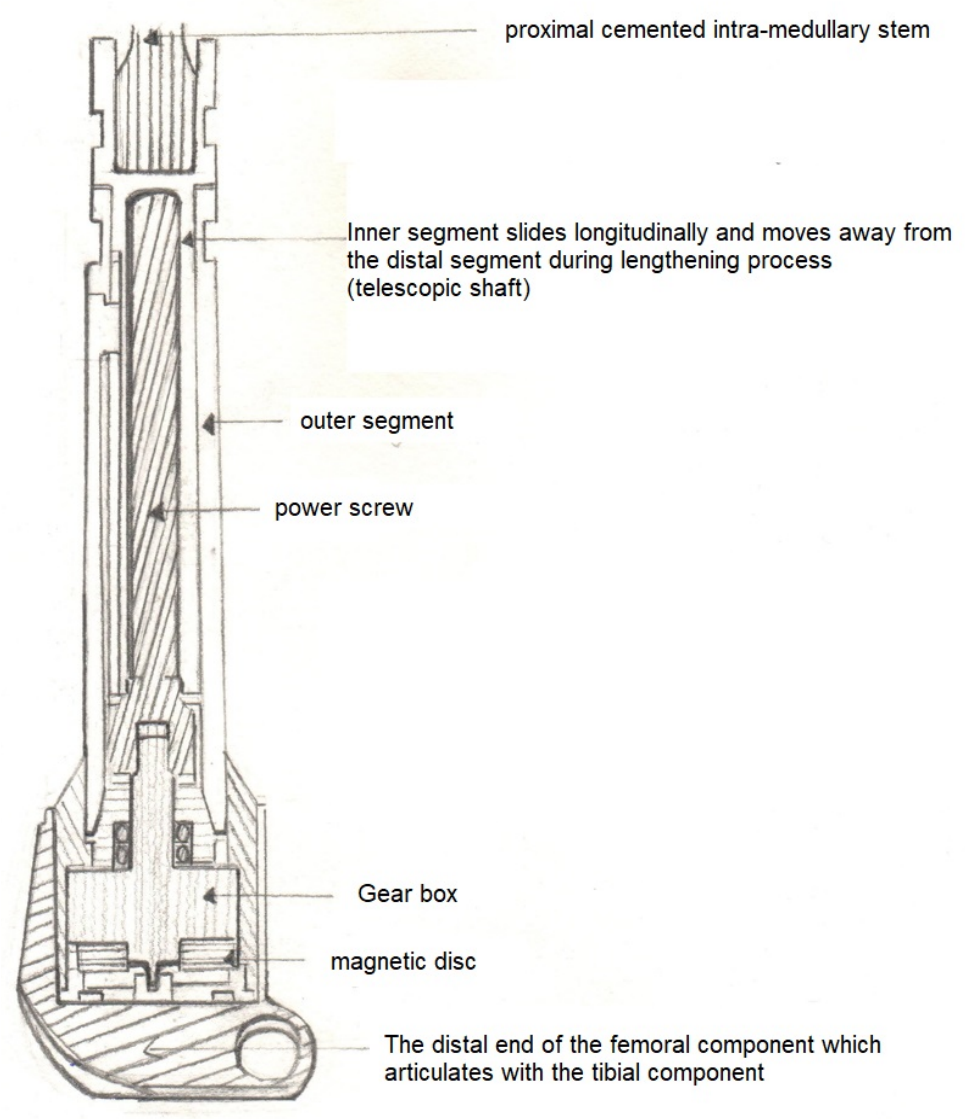

Figure 1: Stanmore Expandible Prosthesis
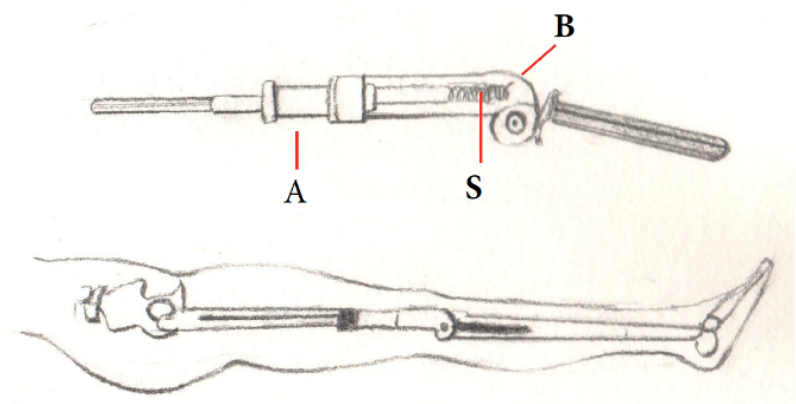

\section{A: Titanium tube \\ B: Polymeric tube \\ S: Spring}

Figure 2: Phenix Expandible Prosthesis 

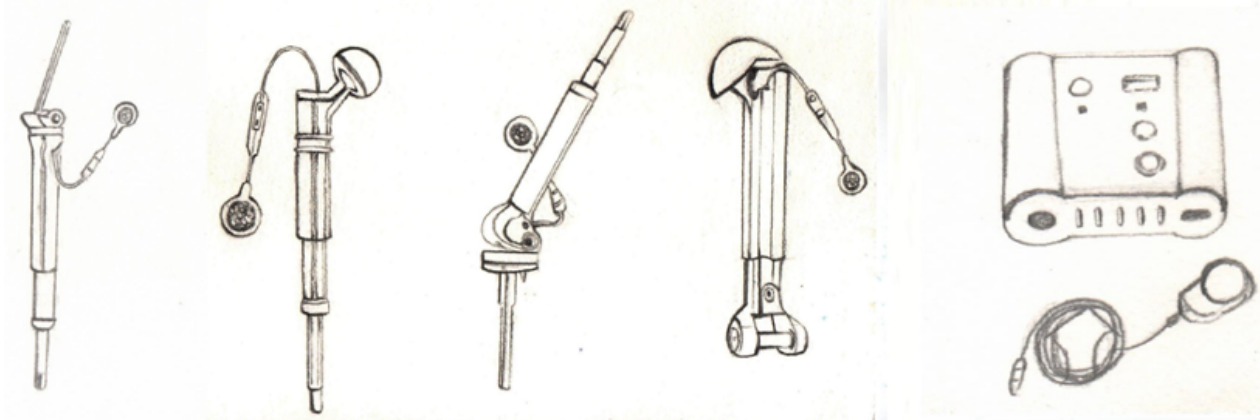

Figure 3: MUTARS Xpand Prosthesis

\section{Conclusion}

The optimal choice of surgical reconstruction following extremity tumor resection in pediatric population remains an enigma. However, the use of endoprosthesis shows good functional outcomes and patient acceptance in addition to reduced rates of amputation following the index surgery. They are associated with high rates of failure and multiple surgeries but these rates are similar to those in the adult population undergoing endoprosthetic reconstruction. The limited availability and high cost also limit the use of expanding endoprosthesis, particularly in low-income countries. In conclusion, endoprosthesis provide a good option for reconstruction in the pediatric population but the long course of follow up and multiple procedures required need to be well understood by the child, parents and the treating surgeon.

\section{Funding}

The authors state that they have not received any funding towards this.

\section{Conflict of Interest}

The authors state that there is no conflict of interest.

\section{References}

1. Campanacci M, Bacci G, Bertoni F, et al. The treatment of osteosarcoma of the extremities: twenty year's experience at the Istituto Ortopedico Rizzoli. Cancer. 1981; 48(7): 1569-1581.

2. Price $\mathrm{CH}$, Zhuber $\mathrm{K}$, Salzer-Kuntschik M, et al. Osteosarcoma in children. A study of 125 cases. J Bone Joint Surg Br. 1975; 57(3): 341345 .

3. Campanacci M, Cervellati G. Osteosarcoma: A review of 345 cases. Ital J Orthop Traumatol. 1975; 1(1): 5-22.

4. van Oosterom AT, Voûte PA, Taminiau AH, et al. Combination chemotherapy preceding surgery in osteogenic sarcoma. Prog Clin Biol Res. 1985; 201: 53-57.

5. Rosen G, Caparros B, Nirenberg A, et al. Ewing's sarcoma: ten-year experience with adjuvant chemotherapy. Cancer. 1981; 47(9): 22042213.
6. Copeland MM, Sutow WW. Osteogenic sarcoma: the past, present, and future. Int Adv Surg Oncol. 1979; 2: 177-200.

7. Simon MA, Aschliman MA, Thomas N, et al. Limb-salvage treatment versus amputation for osteosarcoma of the distal end of the femur. J Bone Joint Surg Am. 1986; 68(9): 1331-1337.

8. Scales JT. Bone and joint replacement for the preservation of limbs. $\mathrm{Br}$ J Hosp Med. 1983; 30(4): 220-232.

9. Unwin PS, Walker PS. Extendible endoprostheses for the skeletally immature. Clin Orthop Relat Res. 1996; (322): 179-193.

10. Schindler OS, Cannon SR, Briggs TW, et al. Stanmore custom-made extendible distal femoral replacements. Clinical experience in children with primary malignant bone tumours. J Bone Joint Surg Br. 1997; 79(6): 927-937.

11. Gupta A, Meswania J, Pollock R, et al. Non-invasive distal femoral expandable endoprosthesis for limb-salvage surgery in paediatric tumours. J Bone Joint Surg Br. 2006; 88(5): 649-654.

12. Wilkins RM, Soubeiran A. The Phenix expandable prosthesis: early American experience. Clin Orthop Relat Res. 2001; (382): 51-58.

13. Groundland JS, Binitie O. Reconstruction After Tumor Resection in the Growing Child. Orthop Clin North Am. 2016; 47(1): 265-281.

14. Dotan A, Dadia S, Bickels J, et al. Expandable endoprosthesis for limbsparing surgery in children: long-term results. J Child Orthop. 2010; 4(5): 391-400.

15. Ruggieri P, Mavrogenis AF, Pala E, et al. Outcome of expandable prostheses in children. J Pediatr Orthop. 2013; 33(3): 244-53.

16. Schinhan M, Tiefenboeck T, Funovics P, et al. Extendible Prostheses for Children After Resection of Primary Malignant Bone Tumor: Twenty-seven Years of Experience. J Bone Joint Surg Am. 2015; 97(19): 1585-1591.

17. Tsuda Y, Tsoi K, Stevenson JD, et al. Extendable Endoprostheses in Skeletally Immature Patients: A Study of 124 Children Surviving More Than 10 Years After Resection of Bone Sarcomas. J Bone Joint Surg Am. 2020; 102(2): 151-162.

18. Zou C, Zhao Z, Lin $\mathrm{T}$, et al. Long-term outcomes of limb salvage treatment with custom-made extendible endoprosthesis for bone sarcoma around the knee in children. J Orthop Surg Res. 2020; 15(1): 14.

19. Groundland JS, Ambler SB, Houskamp LD, et al. Surgical and Functional Outcomes After Limb-Preservation Surgery for Tumor in Pediatric Patients: A Systematic Review. JBJS Rev. 2016; 4(2): 01874474-201602000-00002. 